\title{
EDUCATION AND TRAINING An innovative training and recruitment programme in surgery
}

\author{
Authors: Sriharan Sivayoganathan, ${ }^{A *}$ William English ${ }^{\mathrm{B} *}$ and Veeranna Shatkar ${ }^{\mathrm{C}}$
}

\section{Introduction}

Staff shortages and rising locum costs prompted Barking, Havering and Redbridge University Hospitals NHS Trust to design an innovative training pathway for doctors in surgery. The 'Academy of Surgery' is a 2-year structured educational programme with rotations through surgical and emergency care specialties and includes a funded MSc.

\section{Methods}

We recruited 27 doctors over a 2-year period. The first cohort started in October 2018, the second in October 2019. These doctors are heavily supervised in a 2-year programme that aims to prepare them for higher specialty training. They undergo regular assessment and annual review of competencies and progression. They receive regular formal classroom teaching and there are regular sessions to discuss welfare.

Results

Surgical rotas are now fully staffed and not reliant on locum doctors. This has led to significant cost savings. Locum spending in 2017 was $£ 3,856,000$ vs $£ 1,284,000$ in 2020 - a net saving of $£ 1,187,000$ over 2 years.

\section{Conclusion}

This innovative training programme has contributed to full staffing of a number of surgical rotas within our Trust and delivered a large financial saving for the NHS. We hope to expand this work into neighbouring trusts.

KEYWORDS: innovative, recruitment, training programme

DOI:10.7861/fhj.2020-0063

\section{Introduction}

Barking, Havering and Redbridge University Hospitals NHS Trust (BHRUT) is a large provider of acute services in north-east London. It serves a population of over 750,000, operating via two sites,

Authors: ${ }^{A}$ Academy of Surgery year two trainee, Queens Hospital, Romford, UK; B' general surgery registrar, Queens Hospital, Romford, UK and National Bowel Research Centre, London, UK; ' Consultant emergency and colorectal surgeon, Queens Hospital, Romford, UK; *joint first authors
Queen's Hospital and King George Hospital, and employs over 6,500 staff across these sites. The emergency department at Queen's treats over 150,000 patients annually. The bed capacity across both sites amounts to 900 beds. The Trust was placed in financial special measures in February 2018 due to a significant deficit with regards to total expenditure.

In recent years NHS trusts have seen significantly fewer junior doctors entering into core and specialty training programmes. This has led to more gaps within rotas, leading to increased spending on locum doctors and greater strain on departments and on other junior doctors. ${ }^{1}$ Data released from the foundation programme have demonstrated significantly fewer foundation year 2 doctors entering either core or specialist training: in $201171.3 \%$ entered further training compared to $37.7 \%$ in $2018 .{ }^{2}$ Within our department (general and vascular surgery) we experienced significant rota gaps during this period, which put strain on the remaining workforce, led to increased expenditure on locums and potentially put patient safety at risk.

In response we have designed an innovative training pathway to attract doctors wishing to pursue a surgical career: the BHRUT 'Academy of Surgery', a 2-year structured educational programme with an associated fully funded MSc. This was designed and initiated by the surgical division at the BHRUT to recruit, train and retain junior doctors from across the world.

\section{Methods}

We have recruited 27 doctors to this programme. The first cohort started in October 2018 and the second cohort started in October 2019. All medical graduates except for one were recruited internationally (with MBBS or equivalent degrees recognised by the General Medical Council). Most were in the equivalent of foundation year 2 training or had recently completed foundation training. They were recruited into FYequivalent posts or CT1-equivalent posts depending on their previous experience.

Doctors in the programme complete 6 -month rotations in four specialties out of orthopaedic surgery, general surgery, vascular surgery, urology, ENT surgery, the emergency department and critical care. They also attend weekly departmental teaching, monthly faculty teaching and simulation and skills teaching. They are assigned clinical and educational supervisors, are registered on the Intercollegiate Surgical Curriculum Programme (ISCP) website and undergo an annual review of competencies and progression (ARCP) aligned with the core surgical training curriculum. 


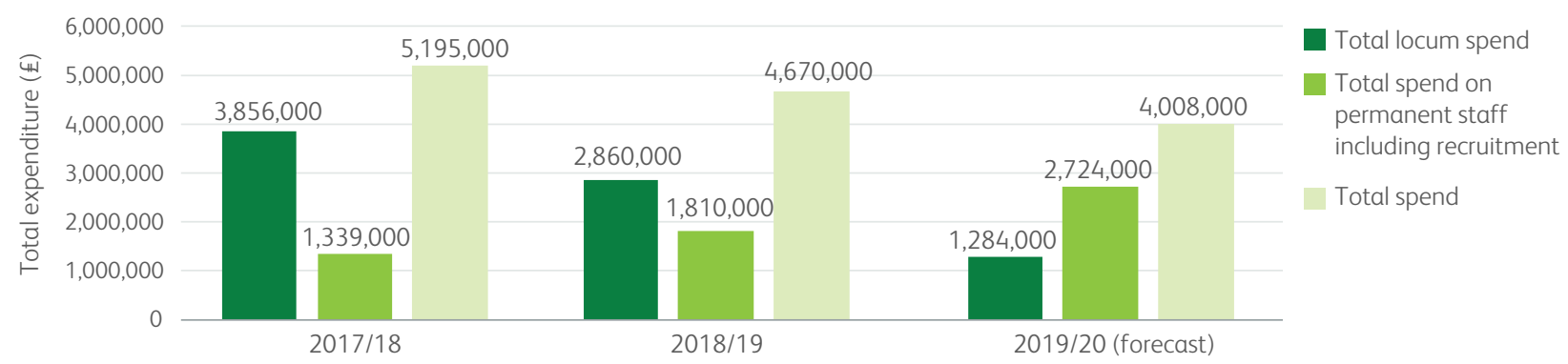

Fig 1. Expenditure per annum on locum and permanent staff.

Concurrently they are funded to complete a MSc degree over the 2-year period from any appropriate institution.

The programme encompasses supervised training activities including outpatient clinics, theatres, ward-based work and an on-call commitment. Trainees are encouraged to complete workbased assessments through the ISCP website and to collect formal feedback as well. Trainees are also allocated a study budget to be used for courses and conferences of interest. Trainees' wellbeing is supported with regular informal meetings to discuss any issues or concerns.

At the end of the programme the trainees are expected to have covered the curriculum for core surgical training, completed their MRCS part A and part B, completed the required number of work-based assessments and to have kept an up to date surgical logbook. If they have met these requirements then they will pass the ARCP and be issued with a 'certificate of completion' from the Academy of Surgery. This should allow them to apply for further surgical training in the UK.

\section{Outcomes}

With the introduction of this programme, we now have a fully staffed rota in most departments and very few rota gaps. This has led to a significant reduction in spending on locum doctors (Fig 1). In 2017 the Trust spent $£ 3,856,000$ on locum doctors; this reduced to $£ 1,284,000$ in 2020 . Spending on permanent staff has increased but by a smaller volume, with the 2017 annual spend being $£ 1,810,000$ and the 2020 spend $£ 2,724,000$. This results in a gross saving of $£ 2,572,000$ and a net saving of $£ 1,187,000$ over the 2 -year period following the introduction of this initiative.

During the 2020 recruitment round, 18 doctors from the Academy of Surgery applied for training posts (nine were successful); two doctors were selected into core surgical training, one doctor was selected into higher surgical training (trauma and orthopaedics), one doctor was selected into radiology training and a further five were successful in securing GP training posts. The remainder are continuing to enhance their portfolios to apply for future recruitment rounds. Two doctors plan to apply for higher surgical training after the completion of MRCS part B, and seven doctors plan to apply for core surgical training. During their time in the Academy, gaining exam-based qualifications was highly encouraged; three trainees completed MRCS parts $A$ and $B$ and $a$ further two trainees have completed MRCS part A.

The programme has been received positively by the Trust. The hospital benefits from a stable and enthusiastic workforce, with the significant increase in permanent doctors within the surgical departments improving communication and making high-quality patient care easier to deliver.

\section{Discussion}

Provision of training has been emphasised as a recruitment and retention tool in medical staffing. ${ }^{3}$ Developing a structured medical education programme with mentorship and coaching results in greater job satisfaction and a significantly upskilled workforce. ${ }^{4}$ Furthermore, it has been demonstrated that recruitment of permanent doctors is more cost-effective for trusts as compared to locum doctors ${ }^{5}$ and helps with strategic workforce planning. ${ }^{6}$

The Academy of Surgery was highlighted as a positive development in the Trust's organisational and workforce strategy report and was subsequently mentioned in the most recent CQC inspection report. The CQC report also describes the Academy of Surgery as being part of the Trust's initiative to recruit and retain a flexible and diverse workforce. ${ }^{7}$ It has also been featured in the Trust workforce recruitment campaign videos (www.youtube.com/ watch?v=bifqDSA_iA4) and website. ${ }^{8}$

In $2018,55.6 \%$ of foundation year 2 doctors were appointed to further posts (including specialty training, locum appointment for training [LAT] and service appointments). Furthermore, $37.7 \%$ of foundation doctors progressed into specialty training. ${ }^{2}$ Though not directly comparable, $50 \%$ of the first cohort ( 9 out of 18 recruits) progressed into a specialty training programme or higher surgical programme, with the remainder continuing to work in service appointments within the Trust.

On the commencement of the programme in October 2018, international medical graduates were ineligible to apply for round 1 of the national recruitment round due to the resident labour market test. Due to the high competition ratio for core surgical training, international medical graduates were rarely able to secure posts in core surgical training, with no posts being advertised in the second round. As a result, the Academy of Surgery offered a certificate equivalent to core surgical competencies at the end of 2 years if demonstrably achieved. As of 6 October 2019, all jobs in the medical profession were included in the UK Shortage occupation list. Thus, all doctors applying for UK-based specialty programme were automatically exempt from the resident labour market test. This enabled the first cohort to apply for core surgical training and specialty training in surgical specialties. The programme has taken into account the recent changes in rules and will revamp its offering at the end of 2 years in October 2020. The second cohort will be applying for the national recruitment round in 2021.

At the beginning of the programme, due to the limitations of applying in the first round, trainees were encouraged to apply for any training programme of interest while taking into account competition ratios, as acceptance rates for international medical 
graduates vary across specialties. With the recent changes to the resident labour market test, the trainees were encouraged to apply for any training programme regardless of competition ratios.

Further review and refinement of the programme is planned in October 2020. We plan to offer 18 months of surgery and a mandatory 6 months in a medical specialty of their choice. This means all the trainees remain eligible to apply for core surgical training (within 18 months of surgical experience) and also have a well-rounded training programme. More robust ARCP procedures and greater focus on research, quality improvement and publications, which was found to be lacking in international medical graduates, has also been planned. The successful candidates who gained entry into specialty training had actively participated in clinical audits and engaged in research, with outputs including posters and oral presentations at conferences. Thus, increased focus on these aspects has been planned and this will be reviewed in trainees' ARCPs by their respective educational and clinical supervisors.

\section{Conclusions}

Over the last 18 months we have demonstrated that the BHRUT Academy of Surgery programme has significantly reduced spending on locum doctors and reduced rota gaps within our department and have received positive feedback from the hospital. Graduates of this programme have successfully applied to formal training in the UK and completed postgraduate exams. We hope to extend our programme into neighbouring NHS trusts, with our Trust remaining as the central hub of the programme. We expect that this will further reduce rota gaps and unnecessary spending while improving patient care.

\section{References}

1 Murray R. The trouble with locums. BMJ 2017;356:j5252.

2 UK Foundation Programme. F2 Career Destinations Report 2018. Available from https://foundationprogramme.nhs.uk/resources/ reports/ [Accessed 12 July 2020].

3 Fahey C, Walker J, Sleigh A. Training can be a recruitment and retention tool for emergency service volunteers. Aust J Emergency Manag 2002;17:3.

4 Eyre HA, Mitchell RD, Milford W et al. Portfolio careers for medical graduates: implications for postgraduate training and workforce planning. Aust Health Rev 2014;38:246-51.

5 Rimmer A. Trusts spend 25 times more on locum fees than on recruiting for permanent posts BMJ 2016:354:14359.

6 White $C$. Trusts are urged to cut spending on locums. BMJ 2012:345:e5977.

7 Care Quality Commission. Barking, Havering and Redbridge University Hospitals NHS Trust: Inspection report. CQC, 2019. www.cqc.org.uk/sites/default/files/new_reports/AAAJ6543.pdf.

8 Guha H. My first year in the Academy of Surgery. www.bhrhospitals.nhs.uk/news/my-first-year-in-the-academy-of-surgery-2161.

Address for correspondence: Mr William English, Queen's Hospital, Rom Valley Way, Romford RM7 0AG, UK.

Email:w.english@nhs.net 